\title{
Exploring Factors Affecting Corporate Performance
}

\author{
Ching-Wen Tung \\ Doctoral Student, National Taiwan University of Science and Technology, Taiwan \\ Lecturer, Department of International Business, Hsing-Wu University, Taiwan
}

Tzu-Tsang Huang

Associate Professor, Department of Finance, Hsing-Wu University, Taiwan

Chiung-Ju Liang

Chair Professor, National Taiwan University of Science and Technology, Taiwan

\begin{abstract}
More than four thousand data of Taiwan listed companies during the period of 2002-2011 are analyzed in this research to explore potential factors that affecting corporate performance. Regression results show that there has been a significant relationship between the ownership structure and corporate performance. Evidence also demonstrates that the indicators of intellectual property have significant impacts on corporate value creation. Findings further indicate that corporate in variant stages of their lifecycle have different performance.
\end{abstract}

KEYWORD: Lifecycle; Intellectual Property; Ownership Structure; Corporate Performance

\section{INTRODUCTION}

Factors affecting corporate performance have always been focuses of the research society as well as practitioners. Managerial behavior, agency costs and ownership structure has been considered as a core issue of corporate governance performance for many years [1-4].

Intellectual capital is becoming more and more important to the corporate performance in the knowledge economic era [5-7]. Intangibles and knowledge management are crucial especial for the value creation of information and communication technology (ICT) industry [8].

Companies in different stages of their lifecycle may have different performance. The stage of the lifecycle of each company may also be an indicator for observing its performance. The five stages of the lifecycle of a corporate can be described as: startup, rapid growth, maturity, decline and rebirth/death [9]. Rapid growth, maturity and decline are the three stages considered in this research that can be defined by the setup years, sales growth, dividend paid, capital expenditure and marketing expenditure.

\section{DATA}

Data consists of 630 listed companies and 4,313 company-years over the time span of 2002-2011 that are collected from Taiwan Economical Journal, annual reports of listed companies by Taiwan Stock Exchange Office and the other public disclosed sources. The aforementioned data are categorized into two industry groups namely traditional industry and high tech industry. High tech industry is loosely defined in this research including industries of integrated circuit, computer and accessories, communication, optical electronic, precision machinery and biotechnology.

\section{VARIABLES AND DEFINITIONS}

All variables and their notations as well as definitions are depicted in Table 1. Corporate performance is the focus and the only dependent variable of this research. In order to compare with the companies within the same industry group, a corporate performance (IRA) is defined as the difference between the return on asset (ROA) of each individual company and the industry average.

Three types of independent variables are included. First of all, ownership structure (OWN) is defined as the ratio of the stocks owned by the board members and major management team members.

Second type of the independent variables is the indicators for the intellectual capitals. Many potential intellectual capital indicators have been discussed in previous researches[10-12]. To simplify the variables, an analytical hierarchical process (AHP) suggested by Saaty [13-15] is applied in this research. Four top most important indicators namely, research and development expenditure (RS), capital expenditure (CA), marketing expenditure (MK) and asset increase (AS) screened by the aforementioned AHP are included in our analyses. 
Table 1. Notation and Definition of Variables

\begin{tabular}{|c|c|c|}
\hline Notation & Variable & Definition of Variable \\
\hline IRA & $\begin{array}{l}\text { Individual Return } \\
\text { on Asset compare } \\
\text { to the industry }\end{array}$ & $\begin{array}{l}\text { Difference between the return } \\
\text { on asset (ROA) of each } \\
\text { individual company and the } \\
\text { industry average }\end{array}$ \\
\hline OWN & $\begin{array}{l}\text { Ownership } \\
\text { Structure }\end{array}$ & $\begin{array}{l}\text { Managerial ownership: } \\
\text { percentage of the stock share } \\
\text { owned by major management }\end{array}$ \\
\hline $\mathrm{RS}$ & Research Spending & $\begin{array}{l}\text { R\&D expenditure / total } \\
\text { revenue }\end{array}$ \\
\hline $\mathrm{CA}$ & Capital Asset & \begin{tabular}{|lll}
$\begin{array}{l}\text { capital } \\
\text { revenue }\end{array}$ & expenditure / total \\
\end{tabular} \\
\hline MK & Marketing & $\begin{array}{l}\text { marketing expenditure / total } \\
\text { revenue }\end{array}$ \\
\hline AS & Asset growth & asset change / total asset \\
\hline SIZ & Size Factor & $\log _{\mathrm{e}}($ total asset $)$ \\
\hline LIA & $\begin{array}{l}\text { Liability (Debt / } \\
\text { Equity Ratio) }\end{array}$ & $\begin{array}{l}\text { total liability / } \\
\text { stockholder's equity }\end{array}$ \\
\hline
\end{tabular}

\section{REGRESSION MODELS}

Corporate performance is first described as a function of the aforementioned independent variables as formulae (1). A second formulation considering the effect of stock ownership (OWN) multiplied by three intellectual capital indicators RS, $\mathrm{CA}$ and $\mathrm{MK}$ can be shown as formulae (2). Applying the Granger causality test, the third formulae (3) is setup to test the cause-effect relationship between stock ownership and corporate performance.

$I R A=\beta_{\sigma}+\beta_{I}(O W N)+\beta_{2}(O W N)^{2}+\beta_{3}(R S)+\beta_{4}(C A)+\beta_{5}(M K)+\beta_{6}(S$ $I Z)+\beta_{7}(L I A)+\beta_{8}(A S)+\varepsilon$

$I R A=\beta_{0}+\beta_{I}(O W N)+\beta_{2}(O W N)^{2}+\beta_{3}(O W N) *(R S)+\beta_{4}(O W N) *(C A$ )$+\beta_{5}(O W N) *(M K)+\beta_{6}(S I Z)+\beta_{\lambda}(L I A)+\beta_{8}(A S)+\varepsilon$

$I R A=\beta_{0}+\beta_{1}(O W N(-1))+\beta_{2}(O W N(-1))^{2}+\beta_{3}(O W N(-1)) *(R S(-$ 1)) $+\beta_{4}(O W N(-1)) *(C A(-1))+\beta_{5}(O W N(-1)) *(M K(-$

1) $)+\beta_{6}(S I Z)+\beta_{\lambda}(L I A)+\beta_{\delta}(A S)+\varepsilon$

\section{RESULTS AND DISCUSSIONS}

Regression results are listed as Table 2. As expected, results show that managerial stock ownership is significantly related to the corporate performance. Three intellectual capital indicators are also significantly affecting corporate performance. Among them, capital expenditure (CA) has a positive impact on value creation of a company. However, R\&D (RS) and marketing (MK) expenditure have negative impacts on corporate performance that requires further investigation.

Debt / equity ratio (LIA) is significantly related to corporate performance and the negative coefficient matches the pecking order theory. Corporate performance is also significantly related to total asset (SIZ) that implies the larger company had better performance during the observed period of time.

Table 2: Regression Results

\begin{tabular}{|c|c|c|c|c|c|c|c|c|}
\hline \multirow{2}{*}{ Vependent: IRA } & \multicolumn{2}{|c|}{ Total Sample } & \multicolumn{2}{|c|}{ Growing Companies } & \multicolumn{2}{|c|}{ Matured Companies } & \multicolumn{2}{|c|}{ Declining Companies } \\
\hline & \begin{tabular}{|l|} 
pooled \\
OLS
\end{tabular} & $\begin{array}{l}\text { OLS } \\
\text { interaction }\end{array}$ & $\begin{array}{l}\text { pooled } \\
\text { OLS }\end{array}$ & $\begin{array}{l}\text { OLS } \\
\text { interaction }\end{array}$ & $\begin{array}{l}\text { pooled } \\
\text { OLS }\end{array}$ & $\begin{array}{l}\text { OLS } \\
\text { interaction }\end{array}$ & \begin{tabular}{|l} 
pooled \\
OLS
\end{tabular} & $\begin{array}{l}\text { OLS } \\
\text { interaction }\end{array}$ \\
\hline OWN & $\begin{array}{l}0.081 * * * \\
(5.560)\end{array}$ & $\begin{array}{l}0.077 * * * \\
(5.231)\end{array}$ & $\begin{array}{l}0.057 \\
(1.554)\end{array}$ & $\begin{array}{l}0.072 * * \\
(1.979)\end{array}$ & $\begin{array}{l}0.066 * * * \\
(3.716)\end{array}$ & $\begin{array}{l}0.060 * * * \\
(3.341)\end{array}$ & $\begin{array}{l}0.093 * \\
(1.954)\end{array}$ & $\begin{array}{l}0.092 * \\
(1.869)\end{array}$ \\
\hline RS & $\begin{array}{l}-0.008^{*} \\
(-1.868)\end{array}$ & & $\begin{array}{l}-0.085 * * * \\
(-3.173)\end{array}$ & & $\begin{array}{l}-0.004 \\
(-0.975)\end{array}$ & & $\begin{array}{l}-0.052 \\
(-1.468)\end{array}$ & \\
\hline $\mathrm{CA}$ & $\begin{array}{l}0.011 \text { **** } \\
(3.912)\end{array}$ & & $\begin{array}{l}0.009 \\
(1.294)\end{array}$ & & $\begin{array}{l}0.015^{* * *} \\
(4.014)\end{array}$ & & $\begin{array}{l}0.026 * * * \\
(2.709)\end{array}$ & \\
\hline MK & $\begin{array}{l}-0.027 * * * \\
(-4.783)\end{array}$ & & $\begin{array}{l}-0.029 * * * \\
(-2.619)\end{array}$ & & $\begin{array}{l}-0.016 * * \\
(-2.256)\end{array}$ & & $\begin{array}{l}-0.119 * * * \\
(-4.628)\end{array}$ & \\
\hline OWN*RS & & $\begin{array}{l}-0.027 * * \\
(-2.146)\end{array}$ & & $\begin{array}{l}-0.200 * * * * \\
(-3.026)\end{array}$ & & $\begin{array}{l}-0.041 \\
(-0.939)\end{array}$ & & $\begin{array}{l}-0.113 \\
(-1.298)\end{array}$ \\
\hline $\mathrm{OWN} * \mathrm{CA}$ & & $\begin{array}{l}0.021 * * * \\
(2.891)\end{array}$ & & $\begin{array}{l}0.019 \\
(1.285)\end{array}$ & & $\begin{array}{l}0.026 * * * \\
(3.145)\end{array}$ & & $\begin{array}{l}0.067 * * * \\
(2.966)\end{array}$ \\
\hline OWN* MK & & $\begin{array}{l}-0.035 * * * \\
(-3.233)\end{array}$ & & $\begin{array}{l}-0.070 * * \\
(-2.427)\end{array}$ & & $\begin{array}{l}-0.024 \\
(-1.611)\end{array}$ & & $\begin{array}{l}-0.255 * * * \\
(-3.783)\end{array}$ \\
\hline SIZ & $\begin{array}{l}0.002 * * * \\
(4.986)\end{array}$ & $\begin{array}{l}0.013 * * * \\
(4.260)\end{array}$ & $\begin{array}{l}0.001^{*} \\
(1.889)\end{array}$ & $\begin{array}{l}0.001 * \\
(1.828)\end{array}$ & $\begin{array}{l}0.001 * * * \\
(3.181)\end{array}$ & $\begin{array}{l}0.002 * * * \\
(3.361)\end{array}$ & $\begin{array}{l}0.002 * * * \\
(3.299)\end{array}$ & $\begin{array}{l}0.002 * * * \\
(3.267)\end{array}$ \\
\hline LIA & $\begin{array}{l}-0.003 * * * \\
(-9.237)\end{array}$ & $\begin{array}{l}-0.004 * * * \\
(-5.145)\end{array}$ & $\begin{array}{l}-0.003 * * * \\
(-4.223)\end{array}$ & $\begin{array}{l}-0.003 * * * \\
(-4.160)\end{array}$ & $\begin{array}{l}-0.003 * * * \\
(-6.570)\end{array}$ & $\begin{array}{l}-0.003 * * * \\
(-6.444)\end{array}$ & $\begin{array}{l}-0.011 * * * \\
(-6.012)\end{array}$ & $\begin{array}{l}-0.012 * * * \\
(-6.147)\end{array}$ \\
\hline AS & $\begin{array}{l}0.057 * * * \\
(27.967)\end{array}$ & $\begin{array}{l}0.051 * * * \\
(23.560)\end{array}$ & $\begin{array}{l}0.064 * * * \\
(14.287)\end{array}$ & $\begin{array}{l}0.064 * * * \\
(14.300)\end{array}$ & $\begin{array}{l}0.069 * * * \\
(23.857)\end{array}$ & $\begin{array}{l}0.069 * * * \\
(23.786)\end{array}$ & $\begin{array}{l}0.029 * * * \\
(8.070)\end{array}$ & $\begin{array}{l}0.029 * * * \\
(8.207)\end{array}$ \\
\hline Observations & 4,313 & 4,313 & 944 & 944 & 2,514 & 2,514 & 855 & 855 \\
\hline Adjusted R-squared & 0.301 & 0.256 & 0.336 & 0.335 & 0.331 & 0.329 & 0.258 & 0.252 \\
\hline
\end{tabular}

Note: The T-statistics are reported in parentheses below the estimate coefficients in each cell; *,**,*** denote significance at the $10 \%, 5 \%$ and $1 \%$ level, respectively 


\section{CONCLUSIONS}

Using public disclosed data in the period of ten years of time from 2002 to 2011, this research investigated the factors affecting corporate performance. Analytical results show that the managerial stock ownership is significantly related to the corporate performance. The intellectual capital indicators have significant impacts on corporate value creation. Further study is, however required to find out more convincing impacts of each intellectual capital indicator. During the studied period of time, the larger company had performance in Taiwan.

\section{REFERENCES}

[1] Berle, A. A., \& Means, G. G. C. (1932), The modern corporation and private property. Transaction Books.

[2] Jensen, M. C., \& Meckling, W. H. (1976), "Theory of the Firm: Managerial Behavior, Agency Costs and Ownership Structure." Journal of Financial Economics, 3(4), 305-360.

[3] Demsetz, H., \& Villalonga, B. (2001), "Ownership Structure and Corporate Performance." Journal of Corporate Finance, 7(3), 209-233.

[4] Barnhart, S. W., \& Rosenstein, S. (1998), "Board Composition, Managerial Ownership, and Firm Performance: An Empirical Analysis." The Financial Review, 33(4), 1-16.

[5] Bukh, P. N., Larsen, H. T. \& Mouritsen, J. (2001), "Constructing Intellectual Capital Statements." Sc\&inavian Journal of Management, 17(1), 87-108.
[6] Liang, C. J., \& Yao, M. L. (2005), The Value-Relevance of Financial and Nonfinancial Information-Evidence from Taiwan's Information Electronics Industry." Review of Quantitative Finance and Accounting, 24(2), 135-157.

[7] Tseng, C. Y., \& James Goo, Y. J. (2005), "Intellectual Capital and Corporate Value in an Emerging Economy: Empirical Study of Taiwanese Manufacturers." R\&D Management, 35(2), 187-201.

[8] Chan, S. H., Martin, J. D., \& Kensinger, J. W. (1990), "Corporate Research and Development Expenditures and Share Value." Journal of Financial Economics, 26(2), 255276.

[9] Lewis, V. L., \& Churchill, N. C. (1983), "The Five Stages of Small Business Growth." Harvard Business Review, 61(3), 30-50.

[10]Mavridis, D. G. (2005), "Intellectual Capital Performance Determinants and Globalization Status of Greek Listed Firms." Journal of Intellectual Capital, 6(1), 127-140.

[11]Subramaniam, M., \& Youndt, M. A. (2005), "The Influence of Intellectual Capital on the Types of Innovative Capabilities." Academy of Management Journal, 48(3), 450-463.

[12]Kamath, G. B. (2008), "Intellectual capital and corporate performance in Indian pharmaceutical industry." Journal of Intellectual Capital, 9(4), 684-704.

[13]Saaty, T. L. (2005), "The Analytic Hierarchy and Analytic Network Processes for the Measurement of Intangible Criteria and for Decision-Making." In Multiple Criteria Decision Analysis: State of the Art Surveys, 345-405.

[14]Stein, E. W., \& Ahmad, N. (2009), Using the Analytical Hierarchy Process (AHP) to Construct a Measure of the Magnitude of Consequences Component of Moral Intensity." Journal of Business Ethics, 89(3), 391-407.

[15]Podvezko, V. (2009), "Application of AHP Technique." Journal of Business Economics and Management, (2), 181189. 